\title{
Penetration of Bevacizumab into the Iris, Anterior Chamber Angle and Ciliary Body After Intravitreal Injection
}

\author{
Swaantje Peters, ${ }^{1,2}$ Peter Heiduschka, ${ }^{1}$ Karl-Ulrich Bartz-Schmidt ${ }^{1}$ and Ulrich Schraermeyer $^{1}$
}

1. University Eye Hospital, Tübingen; 2. University Eye Hospital, Lübeck

\begin{abstract}
Recently, it was suggested that the anti-vascular endothelial growth factor (VEGF) antibody bevacizumab could be used in the treatment of rubeosis iridis. Therefore, we aimed to trace the penetration of bevacizumab into the anterior chamber after intravitreal injection of the drug. We found that intravitreally injected bevacizumab penetrates well and quickly into the anterior chamber angle, iris and ciliary body. The highest concentration of bevacizumab is present on days one to four after injection, with penetration into the iris appearing to be faster than that into the anterior chamber angle and ciliary body. These findings are consistent with the clinically described regression of iris neovascularisation one to three days after injection. Furthermore, we demonstrated progressive penetration through the tissues of the anterior chamber towards the sclera. Our study showed that the intravitreal application mode is suitable for obtaining an accumulation of bevacizumab throughout the vascularised tissues of the anterior segment. Intravitreal bevacizumab may be used as a supplementary treatment for rubeosis iridis and neovascular glaucoma.
\end{abstract}

\section{Keywords}

Bevacizumab, iris neovascularisation, rubeosis, ciliary body, anterior chamber angle, vascular endothelial growth factor (VEGF), immunohistochemistry, intravitreal injection

Disclosure: The authors have no conflicts of interest to declare.

Received: 19 April 2009 Accepted: 4 May 2009 DOI: 10.17925/EOR.2009.03.01.36

Correspondence: Swaantje Peters, University Eye Hospital Lübeck, Ratzeburger Allee 160, D-23564 Lübeck, Germany. E: Swaantje.Peters@googlemail.com

Rubeosis iridis, the formation of pathological vessels in the iris, is a severe complication of ischaemic retinal diseases such as proliferative diabetic retinopathy or retinal vascular occlusion. The angiogenic cascade starts with a focal or generalised retinal hypoxia, which may appear in diabetic patients due to occlusive changes in the vessel wall and perfusion disturbances, as well as in all kinds of retinal vascular occlusive disease (branch or central retinal vein or artery occlusion).

A variety of pro-angiogenic growth factors are involved in the process of angiogenesis, although vascular endothelial growth factor A (VEGF-A) seems to be the most potent. For example, VEGF is twice as potent as angiopoietin-2 in interrupting tight junctions in retinal endothelial cell monolayers. ${ }^{1}$ Retinal hypoxia upregulates VEGF-A expression, leading to the formation of leaky retinal neovascularisation. The high concentrations of VEGF-A also reach the vitreous and the anterior chamber, and in this way act on the tissues of the anterior segment, leading to the formation of neovascularisation in the chamber angle and the iris. These neovascularisations can reduce visual acuity due to haemorrhages, and they also impair the drainage of aqueous humour in the chamber angle, leading to secondary neovascular glaucoma, which is a severe complication of retinal ischaemic disease. This leads to damage of the optic nerve.

The existing treatment options - such as pan-retinal photocoagulation, cryotherapy or cyclodestructive procedures - often do not generate satisfying results. Recently, use of anti-VEGF antibody bevacizumab in the treatment of rubeosis iridis was suggested.
Bevacizumab is a genetically engineered, humanised, monoclonal antibody (MAb A.4.6.1) against all isoforms of VEGF-A and was approved by the US Food and Drug Administration (FDA) for the adjuvant treatment of metastatic colorectal cancer in 2004. Since 2005 , there have been reports of the 'off-label' use of bevacizumab for ocular neovascular disease. ${ }^{2-4}$ It was found that intravitreal injection of bevacizumab is effective in reducing macular oedema and lesion size of choroidal neovascular membranes, leading to a significant increase or stabilisation of visual acuity. ${ }^{5}$ It was shown that VEGF-induced permeability and proliferation of choroidal endothelial cells can be completely inhibited by bevacizumab. ${ }^{6}$ The number of endothelial cell fenestrations in the choriocapillaris is significantly reduced after intravitreal injection of bevacizumab.

Although retinal and choroidal neovascularisation are the main scope of application for bevacizumab, clinical case reports described a quick regression of leaky iris neovascularisation after intravitreal injection of bevacizumab. ${ }^{8}$ At this time, no experimental work had been published on the effects or time-related distribution of bevacizumab on the vascularised tissues of the anterior segment; neither was it known whether the intravitreal or intracameral application mode was superior.

In terms of the posterior segment, there was intense discussion about whether bevacizumab has the ability to penetrate the retina in order to effect pathological choroidal neovascularisation. The reason for this debate was a study from Mordenti et al. ${ }^{9}$ showing 


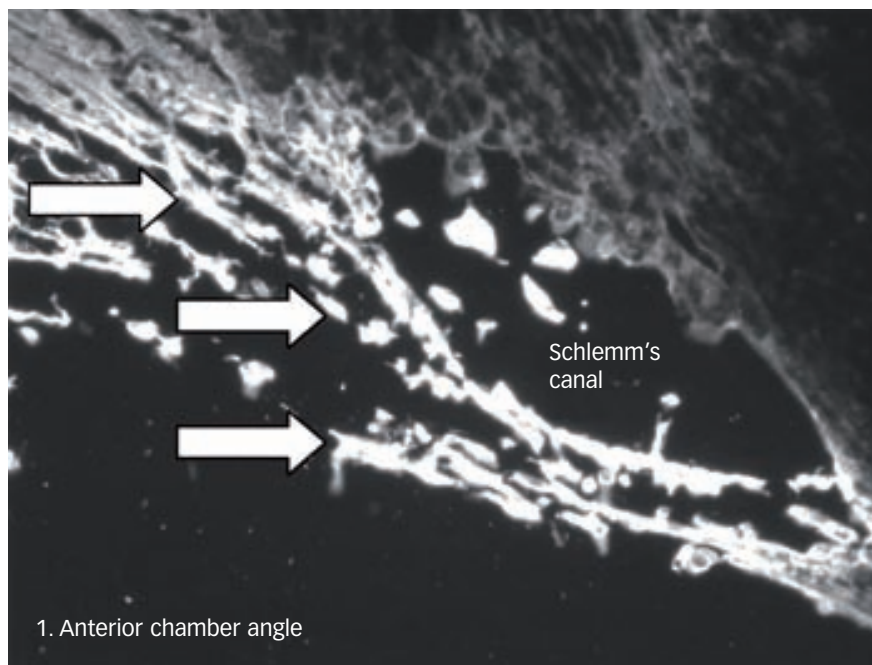

Figure 1: Bevacizumab, shown by white staining, is present in the anterior chamber angle on day one after an intravitreal injection. At this time-point, the immunoreactivity is located at the side of Schlemm's canal that faces the anterior chamber (arrows). On the other side of schlemm's canal, facing the cornea and sclera, there is no positive staining for bevacizumab at this time-point. On day four after the injection, the staining shifts towards the side facing the sclera. On day 14 there is only a faint staining left (not shown).

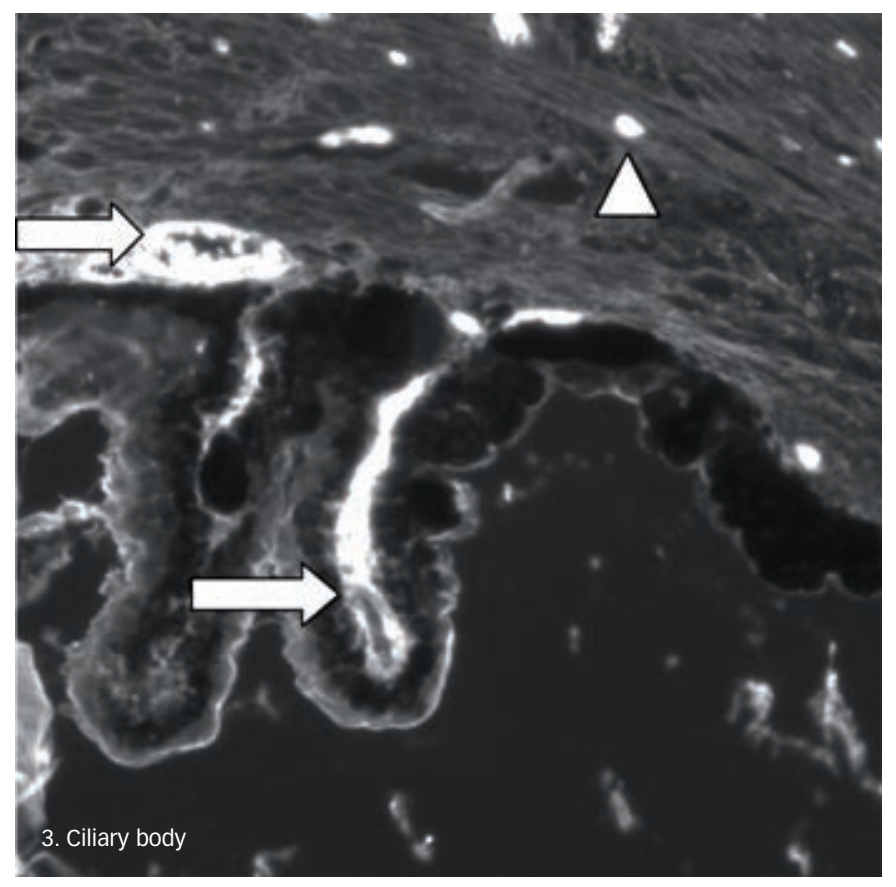

Figure 3: In the ciliary body, a high concentration of bevacizumab is found even on day 14 after injection. It accumulates in the vascular plexus (bottom arrow) of the ciliary processes and is found in the vessel walls of the ciliary body (top arrow). Several vascular lumen are shown to be positive for bevacizumab (arrowhead).

that trastuzumab, a full-length antibody similar to bevacizumab, would be too large to penetrate all retinal layers. Therefore, it was believed that bevacizumab would not be able to penetrate the retina, which led to the development of ranibizumab, an antibody fragment against VEGF-A. However, clinical reports on the intravitreal use of bevacizumab for choroidal neovascularisation first appeared in 2005 and 2006, showing excellent success in the treatment of choroidal neovascularisation..$^{2-4}$ This finding led our study group to test the ability of bevacizumab to penetrate the retina after an intravitreal injection into the primate eye,$^{10}$ and we found good penetration through all retinal layers within the first two weeks after injection.

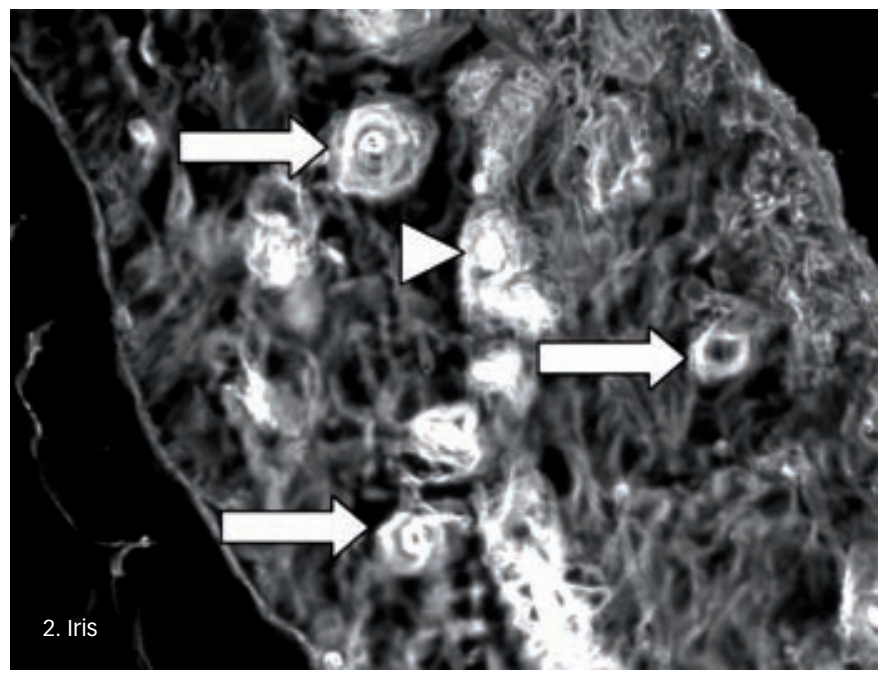

Figure 2: In the iris, the highest concentration of bevacizumab is present on day one after injection. The most intense staining is observed in the blood vessel walls (arrows) and occasionally in a vascular lumen (arrowhead). Bevacizumab staining in the iris diminishes from day four to day 14 after injection (not shown).

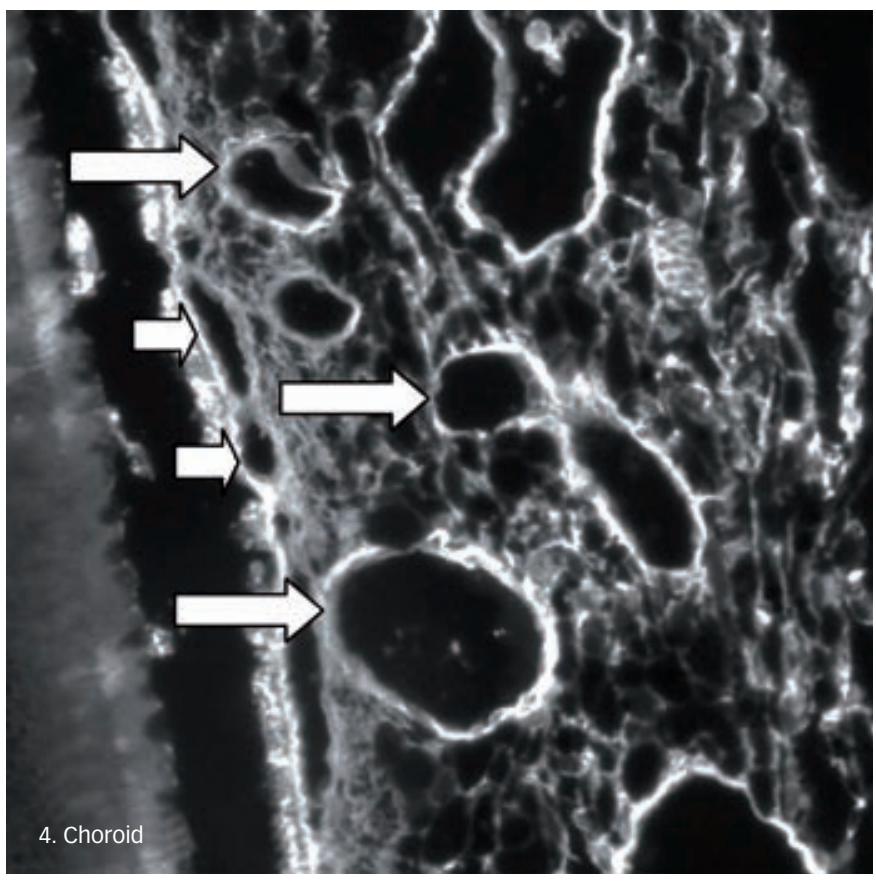

Figure 4: Similar to the tissues of the anterior segment, the most intense staining for bevacizumab is present in the blood vessel walls of the choriocapillaris (small arrows) and the choroid (large arrows).

We also wanted to trace the penetration of bevacizumab in the anterior segment - bearing in mind the question of the penetration of bevacizumab through the retina - even though structures that are not as tight as the retina had to be overcome. ${ }^{11}$ Therefore, we injected $1.25 \mathrm{mg}$ bevacizumab (Avastin ${ }^{\circledR}$, Roche) into the vitreous cavity of primate eyes under general anaesthesia. Control eyes were not treated. The eyes were enucleated on days one, four and 14 and prepared for immunohistochemistry with an antibody to localise bevacizumab.

We found that intravitreally injected bevacizumab penetrates well and quickly into the anterior chamber angle (see Figure 1), the iris 
(see Figure 2) and the ciliary body (see Figure 3). In all of these tissues of the anterior segment, bevacizumab is preferably located in the vessel walls, which are the sites of secretion and binding of VEGF to its receptors on the vascular endothelial cells. ${ }^{12}$ The same

\author{
If the intraocular pressure is high, the use \\ of a local antiglaucomatous treatment or \\ cyclodestructive and filtering surgery \\ becomes necessary.
}

distribution is true for the choriocapillaris (see Figure 4). The highest concentration of bevacizumab is present on days one to four after injection, the penetration into the iris appearing to be faster (day one) than that into the anterior chamber angle and ciliary body (day four).

These findings are consistent with the clinically described regression of iris neovascularisation one to three days after injection, and are also consistent with the observation that the intracameral peak concentration of bevacizumab appears on day three after intravitreal injection. ${ }^{13}$ The intravitreal peak concentration and the anti-oedematous effect on the choriocapillaris were found to be present on day four, obviously paralleling the situation in the anterior chamber. Bevacizumab immunoreactivity in the iris on day one (see Figure 2) is more intense and abundant than in the anterior chamber angle or in the ciliary body, but it then appears to be eliminated more quickly than from the ciliary body. The latter shows an intense positivity for bevacizumab even on day 14 (see Figure 3).

In the chamber angle, there was a time-related distribution of bevacizumab: on day one after injection more intense staining was present on the side of Schlemm's canal facing the anterior chamber (see Figure 1), while on day four the staining was more intense on the exterior side facing the sclera. This finding may reflect a progressive penetration through the tissue from the anterior chamber towards the sclera, paralleling the progressive penetration of bevacizumab through the retinal layers during the first week, as described by Heiduschka et al. ${ }^{10}$
Certain blood vessels showed strong immunoreactivity for bevacizumab in their lumen (see Figures 2 and 3). Assuming a regular circulation, a rapid distribution and subsequent elimination of intravascular bevacizumab would be expected. The selective accumulation of the antibody in certain vessels may be a sign of disturbed circulation in some of the blood vessels, as previously observed in the choriocapillaris. ${ }^{\text {? }}$

We demonstrated that the intravitreal application mode is suitable to obtain an accumulation of bevacizumab throughout the vascularised tissues of the anterior segment. The highest concentration of bevacizumab is seen between days one and four, and it regresses by day 14 . The vessel walls are the target sites for VEGF and therefore show an accumulation of the VEGF antibody bevacizumab in the anterior segment, as well as in the choriocapillaris. Given the persistent intraluminal location of bevacizumab in certain vessels, perfusion disturbances cannot be excluded.

Clinically, bevacizumab may be used as a supplementary treatment for rubeosis iridis and neovascular glaucoma. Although it is not a causative treatment, it has several advantages for the patient. First, the risk of haemorrhages and subsequent deterioration of visual acuity may be reduced. Second, if bevacizumab is used at an early stage of rubeosis, the formation of dense fibrovascular membranes in the chamber angle and resulting neovascular glaucoma may be prevented. If applied at a later stage of the disease, progression may still be inhibited. Nevertheless, additional procedures (panretinal photocoagulation, cryotherapy) to lower VEGF secretion in the hypoxic retina are required. If the intraocular pressure is high, the use of a local antiglaucomatous treatment or cyclodestructive and filtering surgery becomes necessary.

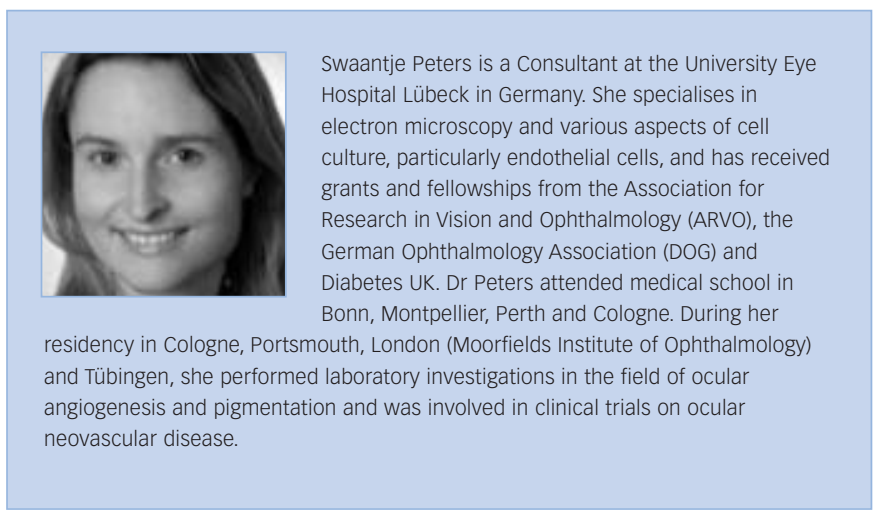

1. Peters $\mathrm{S}$, Cree $\mathbf{I A}$, Alexander $\mathrm{R}$, et al., Angiopoietin Modulation of Vascular Endothelial Growth Factor: Effects on Retinal Endothelial Cell Permeability, Cytokine, 2007;40(2):144-50.

2. Michels S, Rosenfeld PJ, Puliafito CA, et al., Systemic bevacizumab (Avastin) therapy for neovascular agerelated macular degeneration twelve-week results of an uncontrolled open-label clinical study, Ophthalmology, 2005;112(6):1035-47.

3. Reichel E, Intravitreal bevacizumab for choroidal neovascularization and cystoid macular edema: a costeffective treatment?, Ophthalmic Surg Lasers Imaging, 2005;36(4):270-71

4. Rosenfeld PJ, Fung AE, Puliafito CA, Optical coherence tomography findings after an intravitreal injection of bevacizumab (Avastin) for macular edema from central retinal vein occlusion, Ophthalmic Surg Lasers Imaging,
2005;36(4):336-9.

5. Rich RM, Rosenfeld PJ, Puliafito CA, et al., short-term safety and efficacy of intravitreal bevacizumab (Avastin) for neovascular age-related macular degeneration, Retina, 2006;26:495-511.

6. Peters $\mathrm{S}$, Julien $\mathrm{S}$, Heiduschka $\mathrm{P}$, et al., Anti-permeability and anti-proliferative effects of standard and frozen bevacizumab on choroidal endothelial cells, $\mathrm{Br}$ J Ophthalmol, 2007;91(6):827-31.

7. Peters $\mathrm{S}$, Heiduschka $\mathrm{P}$, Julien $\mathrm{S}$, et al., Ultrastructural findings in the primate eye after intravitreal injection of bevacizumab, Am J Ophthalmol, 2007:143(6):995-1002.

8. Grisanti S, Biester S, Peters S, et al., Intracameral bevacizumab for iris rubeosis, Am J Ophthalmol, 2006;142:158-60.

9. Mordenti J, Cuthbertson RA, Ferrara N, et al., Comparisons of the intraocular tissue distribution pharmacokinetics, and safety of 125l-labeled full-length and Fab antibodies in rhesus monkeys following intravitreal administration, Toxicol Pathol, 1999;27:536-44.

10. Heiduschka P, Fietz $\mathrm{H}$, Hofmeister $\mathrm{S}$, et al., Penetration of bevacizumab through the retina after intravitreal injection in monkey, Invest Ophthalmol Vis Sci, 2007;48(6):2814-23.

11. Peters $\mathrm{S}$, Heiduschka $\mathrm{P}$, Julien $\mathrm{S}$, et al., Immunohistochemical localization of intravitreally injected bevacizumab in the anterior chamber angle, iris and ciliary body of the primate eye, Br J Ophthalmol, 2008;92(4): 541-4

12. Simorre-Pinatel $\mathrm{V}$, Guerrin $\mathrm{M}$, Chollet $\mathrm{P}$, et al., VasculotropinVEGF stimulates retinal capillary endothelial cells through an autocrine pathway, Invest Ophthalmol Vis Sci, 1994;35:3393-3400.

13. Bakri SJ, Snyder MR, Reid JM, et al., Pharmacokinetics of intravitreal bevacizumab (Avastin), Ophthalmology, 2007;114: 855-9 